\title{
Quantification of miRNAs by a simple and specific qPCR method
}

Cirera Salicio, Susanna; Busk, Peter K.

Published in:

Methods in Molecular Biology

DOI:

10.1007/978-1-4939-1062-5_7

Publication date:

2014

Document version

Early version, also known as pre-print

Citation for published version (APA):

Cirera Salicio, S., \& Busk, P. K. (2014). Quantification of miRNAs by a simple and specific qPCR method.

Methods in Molecular Biology, 1182, 73-81. https://doi.org/10.1007/978-1-4939-1062-5_7 


\title{
Chapter 7
}

\section{Quantification of miRNAs by a Simple and Specific qPCR Method}

\author{
Susanna Cirera and Peter K. Busk
}

\begin{abstract}
MicroRNAs (miRNAs) are powerful regulators of gene expression at posttranscriptional level and play important roles in many biological processes and in disease. The rapid pace of the emerging field of miRNAs has opened new avenues for development of techniques to quantitatively determine miRNA expression levels in different systems.

In this chapter we describe a PCR method for quantification of miRNAs based on a single reverse transcription reaction for all miRNAs combined with real-time PCR with two miRNA-specific DNA primers. This method quantifies synthetic templates over eight orders of magnitude and successfully discriminates miRNAs that differ by one single nucleotide. Due to the usage of DNA primers this method allows higher amplification efficiencies than a similar method based on locked nucleic acid-spiked primers. The high efficiency translates into higher sensitivity and precision in miRNA quantification. Furthermore, the method is easy to perform with common laboratory reagents, which allows miRNA quantification at low cost.
\end{abstract}

Key words MicroRNA, qPCR, DNA primers, Specificity, Quantification, Noncoding RNA

\section{Introduction}

MicroRNAs (miRNAs) are important regulators of many cellular processes in a broad range of organisms. Due to the important biological role of miRNAs there is a high interest in measuring their expression levels and determine their biological functions in many physiological scenarios. Furthermore, miRNAs have been involved in many disease processes and quantification of their expression can readily contribute to improving diagnostics and prognostics of many human diseases. The presence of stable miRNAs in blood holds great promise as a diagnostic tool for human disease.

The three most commonly used platforms for profiling miRNAs are high-throughput sequencing (miRNA), microarray profiling, and reverse transcription quantitative PCR (RT qPCR). 
The latter method is used alone and for validating data obtained with the other two techniques.

Accurate measurement of miRNAs by qPCR has some inherent challenges. One of them is the design of PCR primers because miRNAs are only around 22 bases long, which is about the size of a conventional PCR primer. Several methods have been developed to overcome this problem. Chen and co-workers [1] developed a stem-loop RT-qPCR in which reverse transcription is done with a special loop primer followed by a PCR with one specific primer and a universal primer. The PCR product is detected with a TaqMan probe. Unfortunately, this method does not allow control of the specificity of the assay by melting curve analysis.

A recently published method based on circularization of the miRNA also depends on a specific primer for reverse transcription [2]. This method uses RNA ligase, which is sensitive to sequence bias.

Another way to perform qPCR on miRNAs is to add a poly(A) tail to the miRNA sequence and use an anchored poly $(\mathrm{T})$ primer for reverse transcription [3]. This method has the advantage that one reverse transcription reaction per sample is sufficient to measure expression of all miRNAs. Subsequently, PCR is performed with a miRNA-specific primer and a universal primer. However, this method relies on only one specific primer for PCR and there is no detection of a sequence-specific probe. In addition, the assays are difficult to design and often the specificity of the PCR is low [4]. An important drawback of this method is the difficulty to discriminate between members of a miRNA family that differ by only one or a few nucleotides.

Universal RT miRNA PCR combines the benefits of a universal RT reaction with the specificity of two miRNA-specific PCR primers [5]. The PCR product is detected with SYBR Green dye, and the specificity of the assay can be assessed by melting curve analysis. The method relies on poly(A) tailing of the miRNAs followed by reverse transcription with an anchored poly $(\mathrm{T})$ primer. The high specificity of this assay arises from the use of two specific primers for PCR. Originally, it was thought that it was necessary to spike the primers with locked nucleic acid (LNA) [5]. However, later it was found that LNA-spiked primers often lead to low amplification efficiency and DNA primers perform better than LNA primers in this assay and are easier to design [6].

In this chapter we describe how to measure miRNA expression by miRNA-specific qPCR. The protocol consists of reverse transcription with a poly $(\mathrm{T})$ adaptor primer followed by qPCR with two miRNA-specific DNA primers (Fig. 1) and includes instructions on PCR primer design. The method has all the benefits regarding freedom of primer design and specificity of the LNAbased method but requires normal DNA primers, which lowers the cost and makes the method accessible to everybody. 
1

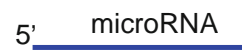

2

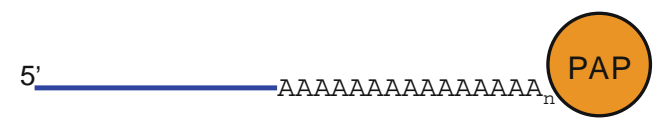

3
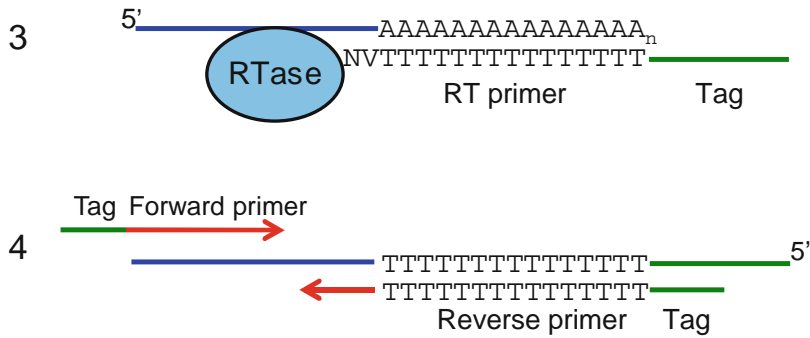

Fig. 1 Scheme of miRNA specific qPCR 1. Start with total PNAor purified miRNA 2. Use poly (A) polymerase to incorporate a tail of adenosines. 3. Make cDNA with reverse transcriptase, RT primer, and dNIPs. Steps 2 and 3 are performed simultaneously in one reaction. 4. Perform real-time PCR with two miPNA specific primers. PAP Poly(A) polymerase, RTase MuLV reverse transcriptase. Modified from Balcells et al. [6]

\section{Materials}

2.1 Reverse

Transcription
All materials and samples should be handled with gloves to avoid contamination with RNases and with nucleic acids that can lead to false-positive results.

All materials should be kept on ice unless indicated otherwise.

1. Poly(A) polymerase: E. coli poly(A) polymerase $5,000 \mathrm{U} / \mathrm{ml}$ (New England Biolabs, USA).

2. Reaction buffer: $10 \times$ E. coli poly $(A)$ polymerase buffer (included with the E. coli poly(A) polymerase).

3. Reverse transcriptase: M-MuLV Reverse transcriptase $200,000 \mathrm{U} / \mathrm{ml}$ (New England Biolabs, USA).

4. $1 \mathrm{mM}$ RNase-free adenosine $5^{\prime}$-triphosphate (ATP): Make it by mixing 1 part $10 \mathrm{mM}$ ATP (included with the E. coli poly(A) polymerase) with 9 parts of RNase-free water.

5. $1 \mathrm{mM}$ RNase-free dNTP mix: Make it by 100 times dilution of a mix of $100 \mathrm{mM}$ deoxy-ATP (dATP), $100 \mathrm{mM}$ deoxy-Cytosine $5^{\prime}$-triphosphate (dCTP), $100 \mathrm{mM}$ deoxy-Guanosine $5^{\prime}$-triphosphate (dGTP), and $100 \mathrm{mM}$ deoxy-Thymidine $5^{\prime}$-triphosphate (dTTP) (Stratagene, USA) with RNase-free water. 
2.2 CPCRReaction

6. RT-primer: $10 \mu \mathrm{M} \quad 5^{\prime}$-CAGGTCCAGTTTTTTTTTTTTT TTVN, where V is A, C, and G and N is A, C, G, and T (Sigma, UK). Order as desalted primer, and dissolve and dilute in RNase-free water or TE.

7. RNase-free water.

8. RNase-free TE (optional): $10 \mathrm{mM}$ Tris- $\mathrm{HCl}, \mathrm{pH} 8.0,1 \mathrm{mM}$ EDTA (see Note $\mathbf{1}$ ).

9. Positive controls (optional): RNA positive controls are made as synthetic RNAs with the same sequence as the miRNA (see Note 2).

10. MS2 RNA (optional, Roche Applied Science) or other carrier RNA to protect the RNA in dilute samples (see Note 2 ).

1. MiR-specific PCR primers: Primers are designed as described in Subheading 3. They should be desalted after synthesis and dissolved and diluted to $10 \mu \mathrm{M}$ in water or TE.

2. PCR mix: Quantifast SYBR Green PCR kit (Qiagen) and Brilliant III Ultra-Fast QPCR Master Mix (Agilent) perform well, but other master mixes may also work (see Note 3).

3. Positive controls for PCR (optional): Synthetic, positive control DNA templates are designed with the sequence 5 '-CAGGTCCAG $\left(T_{15}\right)$ followed by the sequence complementary to the miRNA. This sequence is identical to the DNA sequence generated by the reverse transcription step (Fig. 1) (see Note 4).

4. RNase-free water.

5. RNase-free TE (optional): $10 \mathrm{mM}$ Tris- $\mathrm{HCl}, \mathrm{pH} 8.0,1 \mathrm{mM}$ EDTA.

6. Salmon sperm DNA (optional; Sigma, USA): Use it as a carrier DNA for dilution of synthetic templates (see Note 4).

\section{Methods}

\subsection{Poly (A) Tailing and Reverse \\ Transcription}

1. Mix on ice ( see Note 5):
(a) $1 \mu \mathrm{l} 10 \times$ Reaction buffer E. coli poly(A) polymerase.
(b) 1 Hl $1 \mathrm{mM}$ ATP.
(c) $1 \mu \mathrm{l} 10 \mu \mathrm{M}$ RT primer 5'-CAGGTCCAGTTTTTTTT TTTTTTTVN.
(d) $1 \mu \mathrm{dNTP}$ mix ( $1 \mathrm{mM}$ dATP, $1 \mathrm{mM}$ dCTP, 1 mM dGTP, $1 \mathrm{mM}$ dTTP).
(e) $0.5 \mu \mathrm{l}(200 \mathrm{U} / \mu \mathrm{l}) \mathrm{M}-\mathrm{MuLV}$ reverse transcriptase.
(f) $0.2 \mu \mathrm{l}$ E. coli poly (A) polymerase $5,000 \mathrm{U} / \mathrm{ml}$. 
3.2 QPCR Primer Design
2. Add the RNA sample (see Note 6), 10 pg to 100 ng (see Note 7), and complete with RNase-free water up to $10 \mu$.

3. Incubate at $42^{\circ} \mathrm{C}$ for $1 \mathrm{~h}$.

4. Inactivate the reaction by heating at $95^{\circ} \mathrm{C}$ for $5 \mathrm{~min}$.

5. The resulting cDNA may be diluted $4-8$ times in RNase-free water before being used in qPCR (see Note 8).

Design miR-specific primers according to the previously described rules [6]. They can be made with the software miRprimer [7] or manually by following these steps:

1. Delete all A's from the 3' end of the miRNA sequence until the most $3^{\prime}$ base is $C, G$, or $T$ ( see Note 9 ).

2. Make the longest possible forward primer (12-20 bases long) that leaves at least four bases at the $3^{\prime}$ end of the miRNA for design of the reverse primer and where the $3^{\prime}$ end of the primer fulfills as many as possible of the following criteria (see Note 10):

1. The primer includes one $\mathrm{A}$ or $\mathrm{T}$ residue in the two bases closest to the 3' end.

2. The primer includes one to two $\mathrm{A}$ or $\mathrm{T}$ residues in the three bases closest to the $3^{\prime}$ end.

3. The primer includes two to three $\mathrm{A}$ or $\mathrm{T}$ residues in the five bases closest to the 3 ' end.

3. Calculate the Tm by the nearest neighbor method [8] with a $\mathrm{NaCl}$ concentration of $115 \mathrm{mM}$ (see Note 11).

4. If the $\mathrm{Tm}$ of the forward primer is below $59^{\circ} \mathrm{C}$, add the following bases $\mathrm{G}, \mathrm{A}, \mathrm{C}, \mathrm{G}$, and $\mathrm{C}$ to the $5^{\prime}$ end of the primer one at a time until the $\mathrm{Tm}$ is $59^{\circ} \mathrm{C}$ (see Note 12).

5. If the $\mathrm{Tm}$ of the forward primer is above $59^{\circ} \mathrm{C}$, remove bases from the $5^{\prime}$ end one at a time until the $\mathrm{Tm}$ is $59^{\circ} \mathrm{C}$.

6. Choose the longest possible reverse primer ( $4-8$ bases long) that is not complementary to the $3^{\prime}$ end of the forward primer.

7. Choose the reverse primer with the best $3^{\prime}$ end according to criteria 1,2 , and 3 as in step 2 .

8. Add 15 T's at the 5 ' end of the reverse primer.

9. Calculate the $\mathrm{Tm}$ of the reverse primer as in step 4.

10. If the $\mathrm{Tm}$ of the reverse primer is below $59^{\circ} \mathrm{C}$, add the bases G, A, C, C, T, G, G, A, and C to the $5^{\prime}$ end, one at a time, until the $\mathrm{Tm}$ is $59^{\circ} \mathrm{C}$ (see Note 13 ). 
1. Mix on ice for each sample (see Note 14):
(a) $0.25-1 \mu \mathrm{l} 10 \mu \mathrm{M}$ Forward primer (see Note 15).
(b) $0.25-1 \mu \mathrm{l} 10 \mu \mathrm{M}$ Reverse primer (see Note 16).
(c) $5 \mu \mathrm{l} 2 \times$ PCR master mix.
(d) RNase-free water to a final volume of $9 \mu \mathrm{l}$.

2. Aliquot $9 \mu \mathrm{l}$ in PCR plates or tubes on ice or in a cooling block.

3. Add $1 \mu \mathrm{l}$ of diluted cDNA from previous step. Seal the plate or tubes and spin down (see Note 17).

4. Immediately run the real-time PCR (see Note 18): $95{ }^{\circ} \mathrm{C}$ for 5 min followed by 40 cycles of $95{ }^{\circ} \mathrm{C}$ for $10-30 \mathrm{~s}$ and $60{ }^{\circ} \mathrm{C}$ for 30-60 s (see Note 18).

5. Run melting curve analysis from $55^{\circ} \mathrm{C}$ to $99^{\circ} \mathrm{C}$ ( see Note 19).

Analyze results according to the software in the user's qPCR machine or in MS Excel. For a more thorough statistical analysis we recommend GenEx Pro software (Multid Analyses AB).

\section{Notes}

1. The RT primer can be diluted in TE instead of diluting in RNase-free water. The primer is stable for longer time in TE than in water.

2. Positive control miRNAs can be used for controlling the specificity and the efficiency of the reverse transcription. They can be used as spike in the samples or as pure templates diluted to the desired concentration in $2 \mathrm{ng} / \mu \mathrm{l}$ MS2 carrier RNA. RNA oligonucleotides are kept on ice during use and should be stored in aliquots at $-80^{\circ} \mathrm{C}$ to avoid degradation.

3. Solutions containing SYBR Green should be kept in the dark as the dye is degraded by light.

4. Synthetic DNA can be used for measuring the specificity, detection limit, and amplification efficiency of the qPCR reaction but should be handled with care. Positive controls are extremely prone to contaminate the lab. Therefore, positive controls should be purchased from a different oligonucleotide provider than the one that provides the primers and diluted to $10^{6}-10^{8}$ copies per $\mu \mathrm{l}$ in a background of salmon sperm DNA (carrier DNA) in a different lab and using different pipettes than for the RT and PCR samples. We strongly discourage working with synthetic DNA templates if only one set of pipettes is available. However, it can be done if the pipettes are properly decontaminated according to the manufacturer's guidelines after handling the positive controls. Please refer to http://www.pipettecalibration.net/pipette-cleaning.html for some general guidelines. 
5. It is preferable to make a master mix for all the samples to minimize the effect of pipetting inaccuracy between samples. Make a $10 \%$ excess of the master mix. For example make $47 \mu \mathrm{lmix}$ for nine samples. The mix can be scaled up or down for larger or smaller reverse transcription sample volumes, respectively, by changing the amounts of all components proportionally.

6. Several commercial methods are available for total RNA sample preparation. We have tried two of them with success: miRNeasy (Qiagen) and TriReagent ${ }^{\mathrm{TM}}$ (MRC Research Inc.).

miRNeasy is a column-based method that according to the manufacturer purifies RNA molecules down to 18 base pairs long. The problem of this method is that it is necessary to start with small amounts of tissue (50-100 mg), which results in low amounts of purified RNA. This is especially problematic in tissues with low content of RNA, i.e., fat tissues.

TriReagent ${ }^{\mathrm{TM}}$ is a single-step total RNA isolation reagent, which is not based on columns, and, therefore, it is less expensive and allows larger amounts of tissue than miRNeasy resulting in higher yields of pure RNA. The drawback of this method is that it is difficult to completely remove the phenol used for purification. Often small rests of phenol remain in samples isolated with this method resulting in 260/230 ratios below 1.8. However, this is usually not a big issue for samples analyzed by qPCR, although it might be a problem for techniques that require starting from a highly pure RNA sample.

7. The amount of RNA can vary but should not exceed $100 \mathrm{ng} / 10 \mu \mathrm{l}$ reaction as too much RNA can inhibit the reaction. The amount of RNA necessary for detection depends on the concentration of the target. For samples containing less than $1 \mathrm{ng} / \mu \mathrm{RNA}$, add $1-2 \mathrm{ng} / \mu \mathrm{l}$ MS2 carrier RNA to avoid losing the sample by absorption to the walls of plasticware.

8. Dilution of the cDNA depends on the amount of input sample and the expression level of the specific miRNA. To be able to detect miRNAs in cDNA made from minute amounts of total RNA, samples should not be diluted, whereas cDNA made from $100 \mathrm{ng}$ of total RNA can be diluted ten times or more.

9. One or more A residues at the $3^{\prime}$ end of the miRNA will be part of the poly(A) tail added by the poly(A) polymerase. Regardless of the length of the tail, 15 T residues will be incorporated during the reverse transcription as the RT primer is anchored to the first non-A base at the $3^{\prime}$ end of the miRNA (Fig. 1).

10. It is far from always possible to comply with the three criteria 1,2 , and 3. Criterion 1 is more important than criterion 2 , which is more important than criterion 3. 
11. Several web services for calculating the Tm of an oligonucleotide are available (i.e., Integrated DNA Technologies website http://www.idtdna.com/analyzer/Applications/ OligoAnalyzer/). Make sure that the site uses the nearest neighbor model for calculation and that the $\mathrm{NaCl}$ concentration is set to $115 \mathrm{mM}$.

12. The longest possible forward primer is CGCAGN 20 , where " $\mathrm{N}_{20}$ " are 20 miRNA-specific bases and "CGCAG" is a tail sequence that is not complementary to the miRNA (Fig. 1). More bases can be added to the $5^{\prime}$ end of the primer if necessary.

13. The longest possible reverse primer is CAGGTCCAGT ${ }_{15} \mathrm{~N}_{8}$, where " $\mathrm{N}_{8}$ " are eight miRNA-specific bases, " $\mathrm{T}_{15}$ " are $15 \mathrm{~T}$ 's, and "CAGGTCCAG" is a sequence complementary to the tail of the RT primer (Fig. 1). More bases can be added to the $5^{\prime}$ end of the primer if necessary.

14. It is preferable to make a master mix for all the samples to minimize the effect of pipetting inaccuracy between samples. A $10 \%$ excess of mix is recommended; for example make $90 \mu \mathrm{l}$ mix for nine samples. Furthermore, the mix can be scaled up or down for larger or smaller sample volumes by changing the amounts of all components proportionally.

15. Optimal primer concentration depends on the sequences of the primers and miRNA. Lowering the primer concentration can solve problems with unwanted products but may decrease the amplification efficiency, whereas higher primer concentration may give higher efficiency but increases the risk of unwanted products. For most primer sets and applications, any concentration within the range of $250-1,000 \mathrm{nM}$ will work.

16. Spin the tubes or the plates to assure that the samples are located at the bottom of the reaction vessel.

17. Although the PCR samples are placed on ice, start the realtime PCR step as soon as possible after mixing all the reagents because some primer-template combinations create artifacts if they are stored on ice for a prolonged time.

18. The incubation time at the different steps in the PCR depends on the thermocycler and should follow the manufacturer's recommendations, whereas the incubation temperatures are fixed. The Tm of the PCR primers is optimized to an annealing temperature of $60^{\circ} \mathrm{C}$. If it is changed, it may also be necessary to design primers with different Tms or change the primer concentration.

19. The melting curve analysis should give a single peak (the Tm of the PCR product), which is normally located between 70 and $76^{\circ} \mathrm{C}$. However, very AT- or GC-rich templates may result in products with higher or lower Tms. 


\section{References}

1. Chen C, Ridzon DA, Broomer AJ et al (2005) Real-time quantification of microRNAs by stemloop RT-PCR. Nucleic Acids Res 33:e179

2. Kumar P, Johnston BH, Kazakov SA (2011) miR-ID: a novel, circularization-based platform for detection of microRNAs. RNA 17:365-380

3. Shi R, Chiang VL (2005) Facile means for quantifying microRNA expression by real-time PCR. Biotechniques 39:519-525

4. Chugh P, Dittmer DP (2012) Potential pitfalls in microRNA profiling, Wiley interdisciplinary reviews. RNA 3:601-616
5. Busk PK (2010) Method for quantification of small RNA species. WO2010085966(A2)

6. Balcells I, Cirera S, Busk PK (2011) Specific and sensitive quantitative RT-PCR of miRNAs with DNA primers. BMC Biotechnol 11:70

7. Busk PK (2014) A tool for design of primers for microRNA-specific quantitative RT-qPCR. BMC Bioinformatics 15:29

8. SantaLucia J (1998) A unified view of polymer, dumbbell, and oligonucleotide DNA nearestneighbor thermodynamics. Proc Natl Acad Sci U S A 95:1460-1465 